\title{
Autoconceito e função do papel em pacientes com câncer de cabeça/pescoço
}

\author{
Self-concept and role function in patients with head and neck cancer \\ Autoconcepto y función de rol en pacientes con cáncer de cabeza/cuello \\ Luana Nunes Caldin ${ }^{1}$ if https://orcid.org/0000-0001-9649-4081 \\ Luis Angel Cendejas Medina' io nttps://orcid.org/0000-0002-2809-5350 \\ Renan Alves Silva² io https://orcid.org/0000-0002-1825-0097 \\ Livia Moreira Barros ${ }^{3}$ io https://orcid.org/10000-0002-0174-2255 \\ Magda Milleyde de Sousa Lima' ${ }^{1}$ io https://orcid.org/0000-0001-5763-8791 \\ Geórgia Alcântara Alencar Melo' ${ }^{1}$ io https://orcid.org/0000-0002-3886-5646 \\ Nelson Miguel Galindo Neto ${ }^{4}$ io hitps://orcid.org/0000-0002-7003-165X \\ Joselany Áfio Caetano ${ }^{1}$ io hitps://orcid.org/0000-0002-0807-056X
}

Caldini LN, Medina LA, Silvamo citar:

政, Melo GA, et al. Autoconceito e

unção do papel em pacientes com câncer de cabeça/pescoço. Acta Paul Enferm. 2021;34:APE00892.

DOI

http://dx.doi.org/10.37689/actaape/2021A000892

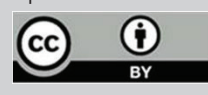

Teoria de enfermagem; Adapta Conceito de papel; Neoplasias de cabeça e pescoço

Keywords

Nursing theory; Adaptation, psychological; Role; Head and neck neoplasms

Descriptores

Teoría de enfermería; Adaptación psicológica; Rol; Neoplasias de cabeza y cuello

Submetido 21 de Abril de 2020

Aceito

17 de Agosto de 2020

Autor correspondente

Joselany Áfio Caetano

E-mail: joselany@ufc.br

\section{Resumo}

Objetivo: Analisar os modos de adaptação de autoconceito e a função do papel em pacientes em tratamento de câncer de cavidade oral.

Métodos: Estudo qualitativo, realizado com pacientes de hospital de referência em câncer, em Fortaleza, CE, Brasil. Utilizou-se da entrevista como técnica de coleta de dados, a partir de instrumento semiestruturado, tendo como base o referencial teórico de Callista Roy. A verificação dos dados ocorreu a partir da análise de conteúdo temática.

Resultados: Emergiram duas categorias temáticas: Sentimentos envolvidos no cotidiano da doença e Perdas de papéis sociais e familiares frente ao câncer de cabeça e pescoço. Revelaram-se declive na autoestima, respostas adaptativas no self-físico, sentimentos negativos no self-pessoal, adaptação positiva no selfpessoal, adaptação positiva, mediada pela crença e religiosidade, na categoria de sentimentos envolvidos. No tocante às perdas de papéis sociais e familiares, identificaram-se alteração de papéis na relação com a família e sociedade, em decorrência da situação trabalhista, e distância entre o convívio da família e necessidade de continuar os ciclos de radioterapia.

Conclusão: Constatou-se que as respostas eficazes no modo de autoconceito se encontram na possibilidade de esclarecer 0 enfoque que deve ser atribuído ao atendimento a essa condição em tela, como forma de promover adaptação à nova condição de vida.

\section{Abstract}

Objective: To analyze self-concept adaptation and role function models in patients with oral cavity cancer.

Methods: This is a qualitative study conducted with patients at a cancer reference hospital in Fortaleza, CE, Brazil. The interview was used as a data collection technique, using a semi-structured instrument, based on Callista Roy's theoretical framework. Data verification occurred based on thematic content analysis.

Results: Two thematic categories emerged: Feelings involved in the daily life with the disease and Loss of social and family roles in the face of head and neck cancer. Slope in self-esteem, adaptive responses in selfphysical, negative feelings in self-personal, positive adaptation in self-personal, positive adaptation, mediated by belief and religiosity were revealed in the category of feelings involved. Concerning loss of social and family roles, changes in roles were identified in the relationship with the family and society, due to the work situation, and the distance between the family and the need to continue radiotherapy cycles. 
Conclusion: It was found that effective responses in the self-concept model are found in the possibility of clarifying the focus that should be attributed to meeting this condition on screen as a way of promoting adaptation to the new condition of life.

\section{Resumen}

Objetivo: Analizar los modos de adaptación de autoconcepto y función de rol en pacientes en tratamiento de cáncer de cavidad oral.

Métodos: Estudio cualitativo, realizado con pacientes de hospital de referencia en cáncer, en Fortaleza, estado de Ceará, Brasil. Se utilizó la entrevista como técnica de recolección de datos, a partir de un instrumento semiestructurado y con base en el marco referencial teórico de Callista Roy. La verificación de datos se realizó a partir del análisis de contenido temático.

Resultados: Surgieron dos categorías temáticas: Sentimientos relacionados con la cotidianidad de la enfermedad y Pérdida de roles sociales y familiares frente al cáncer de cabeza y cuello. Se observó declive de la autoestima, respuestas adaptativas del yo físico, sentimientos negativos del yo personal, adaptación positiva del yo personal, adaptación positiva, a través de las creencias y religiosidad, en la categoría de sentimientos relacionados. En lo que se refiere a la pérdida de roles sociales y familiares, se identificó la alteración de roles en la relación con la familia y la sociedad, como resultado de la situación laboral, y la distancia entre la convivencia de la familia y la necesidad de continuar con los ciclos de radioterapia.

Conclusión: Se constató que las respuestas eficaces del modo de autoconcepto se encuentran en la posibilidad de aclarar el enfoque que debe ser atribuido a la atención de esta condición, como forma de promover la adaptación a la nueva condición de vida.

\section{Introdução}

O câncer é um problema de saúde pública mundial, apresentando 20 milhóes de casos em todo o mundo. No Brasil, estima-se ocorrência de 600 mil novos eventos, dos quais os tumores na regiáo da cabeça e do pescoço sáo os mais frequentes em homens. ${ }^{(1)}$

O Carcinoma de Cabeça e Pescoço (CCP) tem acometimento nos sítios anatômicos do trato aero digestivo superior, que inclui cavidade nasal, seios da face, lábios, glândulas salivares, ossos do complexo craniofacial e laringe, com diferentes causas multifatoriais para o desenvolvimento, entre as mais concorrentes, estão: tabagismo, etilismo, sinergia, além de herança genética, exposição ao sol, por longo período, e papilomavírus humano. ${ }^{(2)} \mathrm{O}$ CCP compreende um grupo heterogêneo de neoplasias malignas, cada uma destas se distingue por ter epidemiologia, gênese, característica patológica, tratamento e prognóstico. Logo, decidiu-se por investigar, neste estudo, o câncer de cavidade oral, por ser o mais recorrente entre os outros tipos. ${ }^{(1)}$

Estima-se, no triênio 2020-2022, 11.180 casos em homens e 4.010 em mulheres. Esses valores correspondem a um risco estimado de 10,69 casos novos a cada 100 mil homens, ocupando a quinta posição. ${ }^{(2)}$ Destarte, o diagnóstico e tratamento têm impacto direto na rotina e qualidade de vida de indivíduos, com alteraçóes na comunicação, deglutição (nutrição), sucção, respiração, deformidades (mutilação), além de não aceitação da imagem facial, problemas emocionais e pouca interação social, em pacientes acometidos na cavidade oral. ${ }^{(3)}$
A cirurgia e a radioterapia se mostram como as medidas de tratamento mais eficazes para estes tipos de cânceres, seguido da quimioterapia, as quais podem prolongar o tempo de recuperação e a adaptação. Porém, qualquer que seja o método escolhido, pode haver sequelas ou efeitos adversos pela agressividade produzida por eles. Neste ínterim, pacientes com câncer de cavidade oral têm alteraçôes físicas e psicossociais complexas, entre estas, destacam-se: depressão, ansiedade, incertezas e falta de esperança, quando comparadas a outros pacientes com diferentes tipos de tumores. ${ }^{(4)}$

Os pacientes com esse tipo de câncer passam por diferentes etapas e estímulos, sendo imprescindível entender a adaptação destes à nova condição de saúde e vida. Assim, o modelo teórico da adaptação da Sister Callista Roy permite aprofundar a compreensão das respostas comportamentais e os problemas de adaptação destes pacientes. Roy descreve que a adaptação se faz necessária para estabilidade da pessoa em termos de saúde, pessoa receptora do cuidado, ambiente e meta de enfermagem. ${ }^{(5)}$ Esse modelo teórico apresenta quatro modos adaptativos: fisiológico, autoconceito, função do papel e interdependência. ${ }^{(6)}$

O modo fisiológico é a forma como a pessoa responde como um ser físico aos incentivos ambientais. ${ }^{(6)}$ Em relação ao modo de autoconceito, enfoca aspectos psicológicos e espirituais da pessoa. Tratase da combinação de convicções e sentimentos em determinado momento. Inclui dois componentes: o self-físico (abrange a sensação e a autoimagem corporal) e o self-pessoal (engloba o self-consistência, o 
self-ideal e o self-ético-moral-espiritual). ${ }^{(6)} \mathrm{O}$ modo de função do papel orienta aspectos sociais relacionados às funçóes que a pessoa ocupa na sociedade, enquanto que o modo de interdependência faz referência a interaçóes relacionadas a dar e receber afeto, respeito e valor. ${ }^{(5)}$

Com isso, apesar de ampla produção científica envolvendo outras teorias metodológicas e modelos de cuidados que englobam aspectos como qualidade de vida, sexualidade, imagem corporal, estratégias de enfrentamento, nota-se escassez de estudos que considere, em particular, os modos de adaptação de autoconceito e a função de papel em pacientes com câncer na cavidade oral. ${ }^{(7)}$ Portanto, torna-se relevante estudos dessa natureza, com intuito de esclarecer o enfoque ao atendimento a pacientes nesses respectivos modos de enfrentar ou adaptar-se à nova condição de vida, bem como de avaliar consistência, natureza das relaçóes, estrutura e suposiçóes dessa teoria em público específico. ${ }^{(7)}$

Diante disso, este estudo deslumbra para Enfermagem um caminho para intervir nos problemas adaptáveis gerados, encaminhando estratégias para açóes do cuidado e capacitando pacientes a desenvolver mecanismos de enfrentamento que possam diminuir as respostas negativas e o desempenho de um novo papel, além de favorecer a vivência com a doença e o tratamento clínico do CPP. Desta forma, objetivou-se analisar os modos de adaptação de autoconceito e a função do papel em pacientes em tratamento de câncer de cavidade oral.

\section{Métodos}

Estudo descritivo, com abordagem qualitativa, realizado em hospital de referência em câncer, localizado na cidade de Fortaleza/CE, Brasil, de maio a junho de 2019.

Os participantes compreenderam 16 pacientes maiores de 18 anos, de ambos os sexos, com diagnósticos de câncer de cavidade oral e em tratamento radioterápico, que residiam em Fortaleza ou em municípios próximos. Excluíram-se os pacientes incapazes de se comunicar verbalmente. O quantitativo de participantes foi definido quando a coleta de dados não produziu novas informaçóes, tornando-se redundantes.

Os dados foram obtidos por meio da técnica de entrevista gravada, subsidiada por instrumento semiestruturado, abordando questôes sociodemográficas (idade, sexo e ocupação) e norteadoras do estudo: o que mudou em sua vida com o diagnóstico de CPP? Como você percebe seu estado de saúde, atualmente?

A coleta de dados ocorreu, inicialmente, com a utilização da lista de agendamento das sessóes radioterápicas. A abordagem do participante aconteceu na sala de espera para sessóes terapêuticas; ao verificar que o paciente se enquadrava nos critérios de inclusão, realizou-se contato de aproximação, apresentação da pesquisa, objetivos, método a ser utilizado e, por fim, o convite para participação no estudo e início da entrevista, de forma individual.

Após coleta dos dados, procedeu-se à transcrição dos depoimentos gravados. Posteriormente, as falas foram submetidas às etapas: pré-análise; exploração do material ou codificação; tratamento dos resultados; inferência e interpretação. ${ }^{(8)}$ Nestas etapas, as unidades de significação foram organizadas em quadros analíticos, sendo contabilizada a recorrência em termos de unidades de registro, dentro de cada entrevista transcrita e entre elas, buscando-se, também, a aderência temática para categorização. Em seguida, realizaram-se inferência e interpretação dos dados, baseados no referencial teórico de Roy. ${ }^{(6)}$

O estudo foi desenvolvido após aprovação do Comitê de Ética em Pesquisa da Universidade Federal do Ceará, de acordo com parecer no 2.926.620 e Certificado de Apresentação para Apreciação Ética 52850416.6.0000.5528/2018. As unidades de registro das falas dos participantes foram identificadas pela vogal E, acrescida do número correspondente à ordem das entrevistas, a fim de preservar o sigilo e o anonimato dos participantes.

\section{Resultados}

Entrevistaram-se 13 homens e três mulheres. As idades dos indivíduos variaram de 35 a 90 anos, com média de 56,93 anos. Com relação à escolaridade, a maioria dos entrevistados possuía baixa escolarida- 
de, com média de 5,61 anos de estudo. No tocante à ocupação, oito estavam afastados das atividades laborais por auxílio doença, seis aposentados e dois desempregados. No concernente à modalidade de tratamento, oito estavam realizando unicamente radioterapia, seis radioterapias associadas à quimioterapia e dois radioterapias subsequente à cirurgia.

Emergiram, neste estudo, duas categoriais temáticas nomeadas: Sentimentos envolvidos no cotidiano da doença e Perdas de papéis sociais e familiares frente ao câncer de cabeça e pescoço.

\section{Sentimentos envolvidos no cotidiano da doença}

Vislumbraram-se, nesta categoria temática, cinco subcategorias, representadas pelos núcleos temáticos: baixa autoestima pelas mudanças da condição clínica no self-físico; sentimentos positivos e respostas adaptativas de enfrentamento da doença e ao tratamento; sentimentos negativos que afetam o modo de adaptação no self-pessoal; respostas positivas no self-pessoal; e soluçóes positivas pautadas na esperança de cura e continuidade da vida.

No tocante à percepção de baixa na autoestima, em virtude das mudanças decorrentes do diagnóstico, da evolução e terapêutica empregada, notaram-se, nas falas, tristeza e insatisfação.

\section{“Estou péssimo”. (E4)}

"Perdi a voz, tive muita dificuldade para me alimentar, beber água, e náo sinto o gosto dos alimentos, eu como para comer porque náo sinto sabor. $E^{\prime}$ isso que me incomoda". (E6)

"Mudou muito, toda a minha vida [...] minha rotina." (E7)

"Não posso fazer nada, até porque eu ceguei e não consigo enxergar. Agora nem posso mais trabalhar, está difícil a vida." (E11)

"É algo instável, às vezes, estou bem, outras vezes, não!" (E14)

Alguns pacientes demonstraram, na subcategoria do self-físico, sentimentos positivos e respostas adaptativas de enfrentamento da doença e ao tratamento em relação à imagem corporal:

"Estou mais magro, perdi muito peso, mas acho que estou melhor. Eu estou me achando com mais coragem." (E3)

"Eu falei para o médico que eu ia ficar como o "Chuck", porque vai tirar osso, olho, céu da boca, operar nariz. Nessa cirurgia, eu tive paralisia facial e fiquei mais horroroso. Não tenho tristeza não. Com relação à cirurgia, eu não tenho problema nenhum, só quero de volta minha saúde." (EG)

"Não mudou nada, só sinto que está duro aqui (acariciando o mento). É uma dureza que até atrapalha para falar." (E13)

Por outro lado, identificaram-se sentimentos negativos que afetaram o modo de adaptação no self-pessoal, como observado nas falas dos entrevistados:

"Ansioso (às vezes); introspectivo; angustiado (mais ou menos); frustrado; nervoso (até que não); eu fico muito sozinha porque eu moro só. Tem dias que eu não quero nem levantar da cama." (E2)

"Triste; não estou muito disposto, pois estou me alimentando ruim." (E3)

"Eu perdi um olho, está vendo? O jeito que tem é me conformar com só um, não está fácil ainda." (E16)

"Sinto até envergonhado. Quando você vai para um lugar, para uma celebração. Eu pelo menos não vou mais para missa porque sei que o povo fica cochichando. É constrangedor!” (E8)

Observaram-se, também, que alguns participantes demonstraram respostas positivas no aspecto geral, o que pode ser considerado modo de adaptação positiva no self-pessoal.

"Espero que dê tudo certo. É o que todo mundo espera, espero que seja rápido." (E1) 
"Praticamente, os tumores sumiram, não tenho mais tumor. Estou bem." (E3)

“(...)isso pesou muito, mexeu comigo, mas agora estou bem. Ando de ônibus, saio, me acostumei, "tiro onda”. Então, minha vida agora é só curtir e ficar curada." (E6)

"Está mudando tudo, me recuperando, a saúde principalmente, ficando melhor com a "radio". Já me sinto vitoriosa. Sabe quando você percebe que está vencendo." (E13)

"Hoje, tento ver minha vida como se não estivesse doente, como uma pessoa normal [...] não é porque estou doente que vou morrer hoje ou vou amanhã. [...] Eu chorei na primeira semana. Mas, depois botei na cabeça que vou viver como se todos os dias fossem o último. [...]. Estou mais próximo dos meus filhos, estou vivendo mais em casa, coisas que não fazia antes. É um lado positivo." (E14)

Os pacientes desenvolveram respostas positivas, pautadas na esperança de cura e continuidade da vida, por intermédio da religiáo e das crenças religiosas, demonstrando que quando encontram apoio, podem ter melhora no autoconceito.

"Estou esperando melhorar, se Deus quiser." (E2)

"Tudo bem, graças a Deus. Eu confio nas escrituras: não tenha medo do que está prestes a sofrer... seja fiel até a morte, e eu lhe darei a coroa da vida." (E3)

"Não, a pessoa fica mais cansada, porque mexe muito dentro da gente. Se Deus quiser vou dar uma melhorada, tenho fé no tratamento, em Deus." (E13)

"Uma hora melhora! Se Deus quiser, estou ainda no começo." (E7)

"Espero a restauração da minha saúde. Uma coisa eu tenho certeza, Deus já me curou, eu vim de lá da minha cidade só para buscar o troféu e só saio daqui com ele." (E15).
$\mathrm{O}$ apego à religiosidade e a crença em Deus influenciam, positivamente, a percepção de pacientes acerca do tratamento, muitas vezes, traduzidas como oportunidade de sobrevivência.

\section{Perdas de papéis sociais e familiares frente ao câncer de cabeça e pescoço}

No que diz respeito ao aspecto social, percebeu-se que diante da situação de crise, houve alteração de papéis na relação com a família e sociedade, demonstrado pelo conflito que quando perpetuado, conduz ao fracasso do papel.

"Não me sinto bem, sendo cuidado pelos outros. Se eu enxergasse seria outra coisa [...] é ruim assim, sem poder trabalhar e fazer nada!" (E11)

"Parei de trabalhar, as pessoas olham diferente para o rosto. Ninguém quer manter um empregado com uma ferida feia dessa. Dai como vou manter minha familia." (E4)

"Não vou poder mais trabalhar e quando operar, não vou ter uma vida normal, vai ter que mudar muitas coisas." (E12)

Observou-se que os participantes apresentaram respostas negativas na função do papel, vislumbradas como distanciamento do papel, em decorrência do tratamento, da distância familiar e necessidade de continuar os ciclos de radioterapia.

"[...] a pessoa se afasta da familia para fazer o tratamento, é muito doloroso [...] as viagens também, tenho que deslocar lá do interior, dai quando volto, meus filhos já estão dormindo." (E3)

"Me afastei da família. Não dá para ficar indo e vindo, é cansativo, chega ser doloroso pela fadiga e pela saudade. Minha rotina é essa [...] Tive que abandonar meu trabalho [...]Fico aqui a semana todinha." (E1)

As respostas, como desveladas nas falas, demonstraram que o trabalho era importante e que a limitação funcional ocasionava frustração e impo- 
tência. Ademais, enfatiza-se que o suporte familiar é decisivo no enfrentamento da doença.

\section{Discussão}

Este estudo se limita por não evidenciar outros estudos qualitativos que explorassem a adaptação do autoconceito e a função de papel em pacientes com CPP. Ainda, a maioria dos participantes não tinha sido submetida a cirurgias plásticas reconstrutoras, mostrando-se como estímulo residual, na modificação das respostas negativas da adaptação. Ademais, cita-se o fato de desconsiderar a rede de apoio na análise dos modos adaptativos como outra restrição desta pesquisa.

Constatou-se que estudos relacionados à Teoria de Roy em indivíduos com câncer de cavidade oral, ainda, são poucos explorados. Isto posto, este estudo poderá contribuir e aprimorar os modos de adaptação, no tocante à consistência, às relaçôes, à estrutura, às suposiçóes, à congruência e significância para prática da enfermagem oncológica, nesse público específico. Assim, é possível intervir nas respostas e nos comportamentos negativos, a fim de promover melhor adaptação, durante diagnóstico, tratamento e acompanhamento pós-radioterapia.

Ao considerar que o câncer é um acontecimento inesperado, desencadeador de inúmeros sentimentos, tanto em clientes quanto familiares, os quais proporcionam sensaçóes desagradáveis, por envolver situação nova, exige-se promoção da adaptação, de forma positiva ou negativa, seja ativa ou passiva. $(9,10)$ Assim, cada indivíduo reage de modo particular, uma vez que são influenciados pelas próprias expectativas, história de vida e vivências cotidianas. Atrelado a este fato, todos os entrevistados relataram sentir-se bem e felizes, antes do adoecimento e das alteraçôes na imagem corporal. ${ }^{(3)}$

Conforme observados nas falas deste estudo, o momento de confirmação da doença pode desencadear sentimentos e reaçóes diferenciadas, como desespero, nervosismo, apreensão e estresse. ${ }^{(11)}$ Esses sentimentos, de acordo com Roy, são fundamentais para geração de respostas adaptativas, processadas por meio de quatro canais: processo perceptivo e infor- mativo, aprendizado, julgamento e emoção. Assim, essa regulação favorece mecanismos a serem construídos ao longo do tratamento do câncer de cavidade oral. Neste sentido, compreender o adoecimento como processo que abrange componentes biológicos, psicológicos, ambientais, sociais e espirituais, é imprescindível para auxiliar o indivíduo, oferecendo cuidados, além dos aspectos puramente técnicos.

No tocante ao modelo de adaptação do autoconceito, observaram-se, nas falas, elementos do ser físico, ser pessoal, ser espiritual. Deste modo, com relação ao self físico, à aparência e ao estado de saúde da pessoa, os tratamentos radioterápico, quimioterápico e cirúrgico podem alterar a autoimagem, pois, frequentemente, causam alteraçóes no órgão e na pele, podendo, em casos mais graves, acarretar o aparecimento de úlceras. ${ }^{(12,13)}$ Ainda, notou-se que as respostas advindas nessa condição são negativas no autoconceito, decorrentes de manifestaçôes clínicas próprias dessa modalidade terapêutica, como a fadiga, exacerbando episódios de angústia, ansiedade e medo frente ao desconhecido. ${ }^{(14)}$

Destarte, essas alteraçóes atingem o sujeito em totalidade e drasticamente nas relaçóes interpessoais, prejudicando a integridade social do indivíduo, corroborando os pressupostos teóricos de Roy. $\mathrm{O}$ aspecto estético exerce papel importante na interação social de indivíduos, sendo que as deformidades faciais parecem causar mais impacto do que outras incapacidades físicas no câncer de cavidade oral. ${ }^{(15)}$ Pacientes com deformidades faciais devem receber apoio psíquico para lidarem com o subsequente impacto negativo na autoestima, além dos frequentes estados de tensão e medo, decorrentes da mutilação, do desconhecimento em relação à doença e da possibilidade de morte.

Estudo aponta que as alteraçóes causadas pelo câncer ou pela cirurgia causam impacto e assombro para quem vê, além do sentimento de exclusão e constrangimento em quem as têm. ${ }^{(16)}$ Assim, observou-se que a localização do tumor determina a vergonha, pois a região de cabeça e pescoço é exposta, dificultando a adaptação de forma positiva, a partir do ser pessoal. A doença se manifesta de forma escancarada, sendo algo que não se pode ocultar dos olhares e julgamentos alheios, motivando os indiví- 
duos a se distanciarem das atividades trabalhistas, conforme visualizado nas falas acerca das perdas de papéis sociais e familiares, frente ao câncer de cabeça e pescoço.

Os pacientes relataram percepção negativa das aparências, o que demonstra resposta ineficaz, no modo de adaptação autoconceito. As mudanças físicas oriundas do tratamento fizeram com que os participantes respondessem, de forma insatisfatória, a essa experiência. Entretanto, verificou-se que a espiritualidade e religiosidade influenciam a disposição e esperança para enfrentamento da doença e manutenção da vida. ${ }^{(17)}$ Deste modo, esses dados corroboram os pressupostos filosóficos apontados pela teórica, em que as pessoas têm relação mútua com o mundo e Deus, além de usaram a capacidade criativa de percepção, iluminação e fé para enfrentar os problemas em busca de adaptaçáo.

No tocante ao modelo de adaptação da função de papéis, verificou-se que, em meio ao tratamento do câncer de cavidade oral, os pacientes encontram dificuldades para adaptar a tal condição, enfrentando perdas observadas pelo conflito, fracasso e distanciamento do papel social que exerciam. Assim, considerando que se constitui necessidade social de se saber quem é em relação aos outros, ${ }^{(18)}$ os papéis são as orientaçóes dadas às pessoas, para que ajam de forma compatível com as funçôes sociais. Portanto, a função social é definida como conjunto de atividades que se espera de uma pessoa, em virtude do papel, e implica determinada posição no espaço social. ${ }^{(19)}$

Nesse contexto, compreende-se que o corpo é uma construção suscetível a mudanças que dependem não apenas do biológico, como também dos significados da vida cotidiana construída pelo meio social. Estudo revelou que pacientes com esse tipo de câncer se sentiram "diferentes", correspondendo a 91,3\%; dezesseis deles referiram tristeza, após alteração da imagem, equivalente a $69,5 \%$, promovendo conflitos que atrapalhavam as relaçóes familiares, sociais e grupais. ${ }^{(3)}$

Em outro estudo, verificou-se potencial relação com as mudanças faciais, visto que o rosto de uma pessoa é a expressão da identidade e quando este se altera, o sujeito se sente com a autoimagem distor- cida. Surge sentimento de constrangimento e preocupação com a avaliação dos outros sobre a forma física real ou imaginária. ${ }^{(10)}$ Corroborando, em outra pesquisa, os entrevistados demonstraram vergonha com relação à imagem, após o aparecimento da doença, já que possuíam alteraçôes físicas aparentes. (20) Diante disto, compreende-se que uma imagem corporal sem alteraçôes físicas é sinônimo de equilíbrio e bem-estar, a qual está relacionada com o sentimento de felicidade e equilíbrio emocional. ${ }^{(21)}$

Houve sentimento de desespero com relação à aparência. Porém, com o tratamento estético recebido, esse sentimento vai sendo substituído pela superação e aceitação, considerado reposta eficaz, já que, para o paciente, a saúde, neste momento, tornou-se o objetivo mais importante. $\mathrm{O}$ fato de revelarem se sentir bem, apesar da aparência, desaponta que estão desenvolvendo mecanismos de enfrentamento que os fazem alcançar respostas positivas e, consequentemente, adaptar-se frente à doença e ao tratamento em $\mathrm{si}^{\left({ }^{(6)}\right.}$

Os comportamentos negativos são impulsionados por concepçôes de inutilidade e perda do papel familiar, decorrente do impedimento de executarem as atividades que socialmente eram obrigaçóes. ${ }^{(22)}$ Isso revela resposta ineficaz com relação à mudança de papel, fazendo com que esses pacientes não se adaptassem nesse respectivo modo.

Assim, a vida do indivíduo é estruturada em torno das funções que têm ou almejam. Porém, a mudança de papéis depende do funcionamento adaptativo, podendo trazer consequências negativas, caso este não consiga mudar. Neste sentido, há necessidade de se perder para reencontrar-se. Há um querer-viver teimoso que dá coerência. Sobreviver ao câncer, visto como nova chance de vida, caracteriza-se como momento oportuno para aprender novos valores, superar as limitaçóes, a perda de papéis, as desfiguraçôes faciais. Enfim, desfrutar vitoriosamente a sobrevida. ${ }^{(23)}$

\section{Conclusão}

Os participantes do estudo revelaram questóes importantes, vivenciadas quanto aos modelos de adap- 
tação de autoconceito e à função de papel, as quais são capazes de influenciar o ajustamento à nova condição de saúde e vida. Alguns revelaram comportamentos ineficazes na autoimagem e no modo de desempenho de papel. No tocante às perdas dos papéis sociais e familiares, que a maioria se mostrou ineficaz, demonstra-se necessidade de atenção maior para conseguir desenvolver adaptação de forma satisfatória. Assim, é relevante a construção de assistência em conjunto, alinhada às peculiaridades econômicas, sociais, educacionais e culturais. Neste contexto, a abordagem integral deve ser enfatizada como modelo de saúde, ressaltando a importância do profissional enfermeiro na identificação dos problemas de adaptação, a fim de promover assistência que visa mecanismos de enfrentamento para o alcance da adaptação.

\section{Colaborações}

Caldini LN, Medina LAC, Silva RA, Lima MMS e Caetano JA contribuíram com concepção do estudo, coleta de dados análise e interpretação dos dados, redação do artigo, revisão crítica relevante e de conteúdo intelectual e aprovação da versão final a ser publicada. Barros LM e Melo GAA colaboraram com revisão crítica relevante e de conteúdo intelectual e aprovação da versão final a ser publicada.

\section{Referências}

1. Instituto Nacional de Câncer (INCA). Estimativa 2020: incidência de câncer no Brasil. Instituto Nacional de Câncer José Alencar Gomes da Silva. Rio de Janeiro: INCA; 2020.

2. Instituto Nacional de Câncer (INCA). Estimativa 2018: Incidência de câncer no Brasil. Instituto Nacional de Câncer José Alencar Gomes da Silva. Rio de Janeiro: INCA; 2018.

3. Formigosa JA, Costa LS, Vasconcelos EV. Social representations of patients with head and neck cancer before the alteration of their body image. Rev Fund Care Online. 2018;10(1):180-9.

4. Hanson-Heath CA, Muller LM, Cunningham MF. Evaluating enhancements to a perioperative nurse liaison program. AORN J. 2016;103(4):414-20.

5. Phillips KD. Modelo de adaptación. In: Alligood MR, Tomey MA, editors. Modelos y teorías de enfermería. 7th ed. Madri: Elsevier; 2011. pp. 335-57.
6. Roy C, Andrews HA. The Roy adaptation model. Lisboa (PT): Instituto Piaget; 2001.

7. Medeiros LP, Souza MB, Sena JF, Melo MD, Costa JW, Costa IK. Roy Adaptation Model: integrative review of studies conducted in the light of the theory. Rev Rene. 2015;16(1):132-40.

8. Bardin L. Análise de conteúdo. São Paulo: Edições 70; 2011.

9. Jungerman I, Toyota J, Montoni NP, Azevedo EH, Guedes RL, Damascena A, et al. Patient Concerns Inventory for head and neck cancer: brazilian cultural adaptation. Rev Assoc Med Bras (1992). 2017;63(4):311-9.

10. Pirola WE, Paiva BS, Barroso EM, Kissane DW, Serrano CV, Paiva CE. Translation and cultural adaptation of the Shame and Stigma Scale (SSS) into Portuguese (Brazil) to evaluate patients with head and neck cancer. Braz J Otorhinolaryngol. 2017;83(6):697-704.

11. Grandizoli MV, Ibiapina IS, Santos R Junior, Garcia VC. Level of hope, anxiety and depression in patients with cancer diagnoses. Arq Ciênc Saúde. 2017;24(3):65-70.

12. Gensheimer MF, Le QT. Adaptive radiotherapy for head and neck cancer: are we ready to put it into routine clinical practice? Oral Oncol. 2018;86:19-24.

13. Olteanu LA, Duprez F, De Neve W, Berwouts D, Vercauteren T, Bauters W, et al. Late mucosal ulcers in dose-escalated adaptive dose-painting treatments for head-and-neck cancer. Acta Oncol. 2018;57(2):262-8.

14. Avelar JM, Nicolussi AC, Toneti BF, Sonobe HM, Sawada NO. Fatigue in patients with head and neck cancer undergoing radiation therapy: a prospective study. Rev Lat Am Enfermagem. 2019;27:e3168.

15. Al Afif A, Uys HK, Taylor SM. Improving aesthetic outcomes after head and neck reconstruction. Curr Opin Otolaryngol Head Neck Surg. 2018;26(4):227-33.

16. Ray E. Head and Neck Reconstructive Surgery. Cancer Treat Res. 2018;174:123-43.

17. Rocha PT, Lima CA, Dias OV, Paiva PA, Rocha JF. The influence of spirituality and religious ness in oncological treatment: perception of the person with câncer. Rev Tendên Enferm Profis. 2016;8(4):2031-6.

18. Ribeiro FF, Marteleto RM. Configuration of social networks to support people with cancer: a view from the perspective of information and communication in health. Rev Bras Cancerol. 2018;64(1):77-85.

19. Grigolatto T, Chagas MH, Trzesniak C, Crippa JA, Hallak JE, Santos $\mathrm{JL}$. The influence of social roles in the quality of life of patients with schizophrenia. Cad Ter Ocup UFSCar. 2014;22(1):195-203.

20. Graboyes EM, Hill EG, Marsh CH, Maurer S, Day TA, Sterba KR. Body image disturbance in surgically treated head and neck cancer patients: a prospective cohort pilot study. Otolaryngol Head Neck Surg. 2019;161(1):105-10.

21. Hanly P, Maguire R, Balfe M, Hyland P, Timmons A, O'Sullivan E, et al. Burden and happiness in head and neck cancer carers: the role of supportive care needs. Support Care Cancer. 2016;24(10):4283-91.

22. Calver L, Tickle A, Moghaddam N, Biswas S. The effect of psychological interventions on quality of life in patients with head and neck cancer: A systematic review and meta-analysis. Eur J Cancer Care (Engl). 2018;27(1):e12789.

23. Mendes PH, Barbosa HÁ, Rodrigues Neto JF, Leite MT, Sampaio $\mathrm{CA}$. Meaning of facial aesthetic sequelae for subjects undergoing surgery for treatment of head and neck cancer. Unimontes Cient. 2017; 19(1):141-52. 